\title{
LA TUTELA, PROMOCIÓN Y AMPLIACIÓN DE LOS DERECHOS LINGÜÍSTICOS COMO FACTOR PROMOTOR DE IDENTIDAD ÉTNICA Y NACIONAL EN PERÚ
}

\author{
PROTECTION, PROMOTION AND EXTENSION OF LANGUAGE \\ RIGHTS AS A PROMOTING FACTOR OF ETHNIC AND NATIONAL \\ IDENTITY IN PERU
}

\section{ICHINÖKAPO, DAKA WAMBORANDA IKAPO OREDN DERECHO LINGÜÍSTICO OROTA DAKA WATONÖPE OREDN WANDARI PERUYO(1)}

\author{
Manuel Bermúdez-Tapia ${ }^{(2)}$ \\ Universidad Privada San Juan Bautista, Perú
}

\begin{abstract}
Resumen: El reconocimiento de la multiculturalidad, plurilingüismo y la condición cultural histórica del Perú es un tema que aún no adquiere la importancia que debiera tener en la agenda política. Prueba de ello es que no existe una Política de Estado, ni políticas públicas, que se complementen en la atención de las comunidades indígenas para generar una tutela de derechos progresista, autónoma y democrática. Las políticas públicas deben evaluarse desde dicha perspectiva de tutela, promoción y ampliación de los derechos porque no se puede construir un país en función de la exclusión de determinados grupos poblacionales.
\end{abstract}

Palabras Clave: Identidad cultural, Derechos colectivos, Identidad nacional, Derechos lingüísticos, Democracia étnica.

Abstract: The recognition of multiculturalism, multilingualism, and the historical cultural condition of Peru is an issue that has not yet acquired the importance it

(1) Traducción: José Antonio Dumas Ramos. Lengua originaria Harakbut.

(2) Abogado por la Pontificia Universidad Católica del Perú. Magister en Derecho, Doctorado en Derecho por la Pontificia Universidad Católica de Argentina. Profesor Investigador de la Universidad Privada San Juan Bautista y profesor de la Facultad de Derecho de la Universidad Nacional Mayor de San Marcos.Contacto: manuel.bermudez@upsjb.edu.pe, mbermudeztapia@gmail.com ORCID: http://orcid. org/0000-0003-1576-9464 
should have on the political agenda. Proof of this is that there is no State Policy, or public policies, that complement each other in the care of indigenous communities to generate a progressive, autonomous and democratic protection of rights. Public policies must be evaluated from this perspective of protection, promotion and expansion of rights because a country cannot be built based on the exclusion of certain population groups.

Keywords: Cultural identity; Collective Rights; National identity; Linguistic rights; Ethnic Democracy.

Nöngnöng'ä wakkanda harabut hömähënë kënta, kënta nöngnöng haatenda hombahapaknë kënta oredn estado'a daka tonöpëhë möhënë, hanhënta weindapi, kënëng öhëpo agenda nacionalyo orota töhëhë möhënë, política de estadopi oro harakbutwata bakaehe kën complenmenta ïhëpo comunidades indigenasta atiende wamänka, kenpa ikapo daka ikai hopudntenda daka tonöpendik'a. Políticas publicas botahënë kën kenpa ikapo protege kandik möhënë itonöpepo evalua kandik, oro harakbut oredn pueblopi vulnerablenda höhënë kënta iwahapo eka, mënpa ikapo orota excluye ikapo dak país mënpa kahe yähët'apo. 


\section{Introducción}

El análisis del Perú resulta sumamente complejo y muy variado. La historia cultural que existe bajo la República es amplia y milenaria (Bravo, 2005). Un pasado histórico que califica al Perú como uno de los diez países "milenarios" en el ámbito mundial porque cumple con algunos elementos que lo identifican como tal. Un pasado histórico con referencias monumentales y una tradición que sobrevive en el tiempo y una continuidad histórica entre las diferentes eras que se han sucedido en el tiempo.

Sin embargo, este pasado histórico se contradice con una realidad nacional muy especial y particular: la población no es uniforme y los visos de exclusión social, económica y cultural resultan extremos que deslegitiman toda acción de uniformidad o unión nacional (Matos Mar, 1969), generando condiciones limitativas para grupos vulnerables, en particular respecto de la tutela de derechos fundamentales, la accesibilidad a servicios públicos y la defensa de sus derechos particulares, esencialmente en el ámbito étnico, cultural, social y económico (Bermúdez-Tapia, 2001, p. 333).

Las poblaciones de origen étnico andino y amazónico, que provienen de comunidades pre existentes a la Colonia y a la República, son el grupo vulnerable que se analiza en el presente texto. Es imperiosa la necesidad de evaluar el modo en el cual se puedan atender sus justas aspiraciones jurídicas y sociales. Ello sólo es posible a través de la defensa de su idioma por cuanto este es el vehículo que permite la materialización de sus derechos, conforme ha sido acreditado en el Tribunal Constitucional en la STC No 00889-2017-PA/TC, caso María Antonia Díaz Cáceres de Tinoco, en el cual se tuteló el derecho de una persona vernáculo hablante ante una acción contra el Estado. ${ }^{(3)}$

Este es un verdadero problema nacional que implica un elemento negativo para un sector poblacional respecto del Estado. Por ello, resulta necesario considerar algunos elementos para modificar este contexto nocivo, que no puede evaluarse en un sentido unidireccional, dado que tiene repercusiones en varios niveles y contextos:

a. En el ámbito individual, implica la evaluación de los derechos de naturaleza individual de una persona que podría tener problemas de comunicación en la ejecución de cualquier actividad ante el Estado, y en mayor medida si se trata de un seguimiento judicial ante un caso en el que se presenta como demandante, demandado, denunciante o denunciado, con lo cual sus derechos se consideran vulnerables.

(3) En este caso se considera que un quechua hablante ha debido ejecutar: a) Un trámite administrativo ante la Municipalidad Provincial de Carhuaz, en Ancash. B) Un trámite judicial ante el Juzgado Mixto de Carhuaz, en Ancash. c) Un segundo trámite judicial ante la Corte Superior de Justicia de Ancash. d) Un tercer trámite judicial ante el Tribunal Constitucional. (Bermúdez-Tapia, 2018, p. 155). 
b. En el ámbito colectivo, el acceso a servicios públicos básicos como la educación o la prestación de servicios médicos o de accesibilidad a determinados bienes o servicios, permite ampliar negativamente las condiciones económicas, sociales y políticas de exclusión.

c. En el ámbito familiar porque, debido al racismo y discriminación, muchas familias vernáculo hablantes optan por no enseñar o preservar sus valores lingüísticos y culturales (Bermúdez-Tapia, 2012, p. 69).

d. En el ámbito colectivo nacional, esencialmente porque la pérdida de una identidad lingüístico cultural afecta a todo el país porque se pierde un elemento que constituye parte de su esencia cultural y con lo cual no se puede cumplir con los valores detallados en el artículo 48 de la Constitución de 1993.

Es sobre estos elementos que se plantea emplear un elemento socialmente disminuido para elevarlo a una condición positiva, promotora y proactiva de las poblaciones étnicas originarias para así integrarlas al desarrollo nacional, en forma sostenible, promocionando y ampliando su propia identidad para que así repercuta positivamente en el desarrollo nacional y además el país logre preservar su tradición cultural originaria (Remy, 2014, p. 15 y ss.).

Un propósito complejo que parte por explicar elementos generales de una nueva configuración, pero que no requiere de reformas estructurales porque existe un contexto normativo constitucional que respalda la ejecución de la presente propuesta.

Para la validación de esta posición utilizamos una metodología que analiza como unidades de evaluación la legislación, el contexto socio-económico y poblacional. El objetivo de la presente investigación es exponer un proyecto de unidad nacional en función a la equiparación social de las poblaciones existentes en el país, las cuales se reconocen antagónicas e inclusive excluyentes entre sí, factor que limita el valor democrático de la vida política nacional.

Para lograr estos objetivos es utilizada una metodología de estudio hermenéutico, cualitativo, con un enfoque sistémico desarrollado en función a elementos de evaluación provenientes de la doctrina constitucional y de la sociología (i), del análisis normativo constitucional (ii) y de la evaluación de la realidad nacional (iii) que son empleados como variables de estudio, siendo el objetivo demostrar que los derechos lingüísticos constituyen una herramienta básica para viabilizar la defensa legal de los derechos de una persona vernáculo hablante, de origen étnico andino o amazónico. ${ }^{(4)}$

(4) El resultado de este trabajo proviene del documento de investigación vinculado al proyecto: "Análisis de la institucionalidad democrática en el Perú" desarrollado en la Facultad de Derecho de la Universidad Privada San Juan Bautista. Proyecto aprobado por Resolución de Vicerrectorado de Investigación No 061-2019-VRI-UPSJB. 


\section{Análisis del contexto de las políticas públicas inclusivas a nivel poblacional}

La población nacional, como elemento de análisis, no es un elemento muy prolijo en el ámbito de las ciencias sociales, referencia que se amplía en el ámbito de las ciencias políticas y del Derecho Constitucional, muy a pesar de que el INEI (2010), en forma constante, ha ejecutado estudios sobre su composición, desarrollo y proyección en el tiempo (INEI, 2010).

La razón de esta situación parte de la continuidad de valores teóricos y conceptuales arraigados en el ámbito doctrinario que han identificado a la "población nacional" como un valor uniforme, estable, constante en el tiempo y políticamente estandarizada. En este sentido Marcial Rubio (2010) en un análisis de la Constitución de 1993, en varios tomos, no ha tomado en cuenta este contexto, pese a que ejecutó una investigación doctoral (Rubio, 2017).

Bajo esta premisa es que los valores de "Estado" y "Nación" de la Europa Napoleónica se han interiorizado y masificado en el ámbito doctrinario nacional y se ha generado la percepción de que la población peruana es una sola y que las diferencias que existen a niveles colectivos no superan el contexto social o cultural, conforme se puede observar en el análisis que ejecuta Enrique Bernales Ballesteros (2013) a la Constitución de 1993.

Un error de la doctrina jurídica es que no logra asumir el hecho de que en el país existen poblaciones étnicamente originarias y que estas no sólo se han preservado, sino que han logrado incorporarse en la población nacional sobre la base del mestizaje (forzado y obligatorio) que la República desde el gobierno de Simón Bolívar (1826) ha promovido (Luna, 1964).

Justificamos nuestra percepción en la regulación normativa constitucional histórica del Perú. La mejor referencia es que el respeto por valores étnico originarios data de la Constitución de 1979 y esto porque los valores democráticos que impregnaban la reforma constitucional de 1978 permitían un desarrollo de derechos individuales sin excluir valores y derechos de naturaleza colectiva y difusa (Bermúdez-Tapia, 2007). Un hito sin precedentes que permiten detallar que recién en esta época es que se tomó en cuenta el valor referencial de una "población diferenciada" tanto por valores sociales, étnicos, culturales como demográficos.

De este modo, durante el desarrollo de una tradición doctrinaria con una visión eurocéntrica es posible detallar que desde 1821 hasta 1980, la identidad étnico cultural no era un valor con una referencia positiva en el desarrollo nacional, factor que incidía en una serie de políticas públicas que enfatizaban la uniformidad de la población, según las siguientes referencias: 
a. Respecto de las políticas promotoras y productivas del país, en particular las que se generaron en la década de los años setenta del siglo pasado, en lo que implica la industria pesquera, no se tomó en cuenta el valor poblacional y particular de las poblaciones migrantes a las ciudades de la costa peruana (Kleeberg, 2001).

En este sentido, la promoción de una industria pesquera nacional no tomó en cuenta al "trabajador" que había sido condicionado a migrar, tanto por acceder a un trabajo que económicamente le permita sobrevivir (i) como para huir de las secuelas de la Reforma Agraria (ii).

b. Lo anteriormente detallado se relaciona también con la promoción de la educación técnica que había sido diseñada para favorecer la industrialización del país, sin tomar en cuenta el contexto social, cultural y étnico de los "estudiantes", porque lo que se buscaba era generar una población económicamente productiva y laboralmente estable frente a la creciente industrialización (Otoya, 1975).

c. No se ejecutó ninguna acción de atenuación del impacto negativo de la Reforma Agraria, en particular a la población que inicialmente había sido "favorecida".

Dicha Reforma Agraria, muy a pesar de sus propósitos iniciales resultó un fracaso económico, social y nacional a gran escala que provocó el quiebre de las condiciones feudales con las cuales económicamente se manejaba el país, pero que a la vez provocó la exclusión de valores de identidad.

La intención de modificar la identificación de "indígena" a "campesino" provocó un mayor daño que el beneficio propuesto porque a partir de la época en que se produce es que los elementos de discriminación y racismo se elevaron en forma desproporcional, factor que hasta la fecha es manifiesto (Huayre, 2012, p. 104 y ss.).

d. Finalmente, en este ámbito de evaluaciones, es posible observar la política educativa nacional, que desde el inicio de la república transitó por valores equivalentes a otras políticas públicas nacionales respecto de las poblaciones originarias, conforme se detalló en el libro Los derechos lingüísticos (Bermúdez-Tapia, 2001):

1. La invisibilización de las poblaciones originarias, especialmente para negar los derechos de dichas comunidades respecto del reconocimiento de castas (derechos vinculados al ámbito de la Teoría de Estado y derechos de sangre por vínculos políticos con la Corona) que formaba parte del contexto colonial impuesto por la Corona Española. 
2. Una negación de las condiciones, características y derechos de las poblaciones indígenas, al nivel de regularse leyes que en la actualidad serían consideradas denigratorias de la dignidad humana. El "impuesto indígena" es una referencia que históricamente constituye un error del Estado peruano que a la fecha no ha sido atendido ni tampoco corregido.

3. La inserción en el modelo cultural, económico, político, social y pedagógico de la sociedad dominante, para que así el valor productivo del "estudiante" permita mejorar la productividad industrial del país. En este punto, téngase en cuenta lo detallado líneas arriba.

4. La asimilación a los valores culturales, sociales, económicos y políticos de la sociedad dominante, excluyéndose los derechos, valores y cultura de las poblaciones originarias.

Con la reforma de la Constitución en 1993, el proceso de inclusión poblacional de las comunidades originarias a la realidad nacional se expuso de modo negligente y negativo. El artículo $149^{\circ}$ de la Constitución parte de un error constitucional y de un sinsentido técnico, por las siguientes razones:

a. Se hicieron "equivalentes" a las comunidades campesinas y a las comunidades nativas.

Un error que no es reconocido en la doctrina constitucional y en la que defiende el pluralismo jurídico, en particular porque las "Comunidades Campesinas" fueron creadas en el gobierno de Juan Velasco Alvarado y las "Comunidades Nativas" tienen un origen prehispánico y previo a la instauración de la República como forma de gobierno.

Error que es ignorado por la doctrina de forma muy notoria que se justifica porque de lo contrario la legitimidad de las Rondas Campesinas, como forma o expresión socio-cultural histórica no se podría sostener en el ámbito normativo y social.

Referencia e incongruencia que no toma en cuenta que las Rondas Campesinas fueron creadas en Chota (Cajamarca) por personas que no eran indígenas (eran mestizos) y que no tenía por objetivo "preservar" actos sociales y culturales históricos porque el único objetivo era la "lucha contra las acciones de los abigeos".

b. Se permitió "validar" las acciones de las Rondas Campesinas y las Rondas Nativas.

Las "Rondas Nativas" en términos objetivos fueron creadas por el gobierno de Alberto Fujimori para luchar contra el terrorismo y luego el narcotráfico. 
Prueba de este detalle referencial es que a la fecha ningún autor propio del pluralismo jurídico o del derecho constitucional señala una referencia histórica de esta "práctica" que no supera la lucha subversiva de los años ochenta del siglo pasado.

La institucionalidad de las "Rondas Campesinas" también es un error material principalmente porque se detalla que son las comunidades campesinas las que definen el modo en el cual ejercerán una "jurisdicción especial", pero en esencia, estas comunidades no son históricas y constituyen una referencia totalmente diferente a las poblaciones originarias.

Conforme lo detallado, los errores materiales en las políticas públicas vinculadas alámbito poblacional, no han sido diseñadas en forma objetiva (BermúdezTapia, 2000, p. 225) y permiten sostener nuestra posición en los siguientes puntos.

\section{Políticas públicas importantes pero insuficientes}

En el ámbito del diseño de políticas públicas promotoras de derechos, inclusivas y proactivas al contexto de poblaciones originarias en el país, no todo es negativo y prueba de ello es que es posible detallar estas referencias:

\subsection{Políticas públicas en el ámbito educativo y pedagógico.}

La mejor referencia tanto normativa como institucional es el desarrollo del artículo $17^{\circ}$ de la Constitución de 1993 en el campo de la promoción de la Educación Bilingüe Intercultural-EBI (Seminario \& Rebaza, 2018, p. 135 y ss.).

Esta es una política que promueve no sólo los valores étnicos culturales y sociales a nivel individual, sino también a nivel colectivo y difuso porque permite preservar, estudiar y ampliar la defensa de los derechos lingüísticos en el ámbito nacional (Bermúdez-Tapia, 2001, p. 34).

3.2 El derecho a un intérprete en el ámbito administrativo, procesal y judicial, en particular para personas hablantes de un idioma diferente al castellano que es la "lengua de trabajo" oficial en el ámbito estatal peruano (Bermúdez-Tapia, 2006, p. 70), se les permite:

a. Ejercer su derecho a una defensa eficaz y eficiente en el ámbito judicial, pre judicial o administrativo.

b. Desarrollar una posición o argumentación acorde a sus propios intereses y derechos, ejerciendo su derecho de "acción" ante una persona, entidad o ante la propia administración pública. 
La mejor referencia en este ámbito está expuesta en la Sentencia del Tribunal Constitucional peruano, STC No 00889-2017-PA/ TC (Tribunal Constitucional, 2018) que detalla la defensa del derecho de una persona a que se le respete y se le ejecute una comunicación formal en su propio idioma.

c. Ejecutar una "comunicación idónea" entre la administración pública y el ciudadano, conforme se puede detallar en la primera sentencia en idioma originario en el país, emitido en aimara, Expediente No 00134-2011-15-2016-JR-PE-01 (Legis, 2018). En el mismo sentido, en el año 2013 con ocasión del Congreso Iberoamericano de Derecho Constitucional, se señalaba la importancia del derecho a ser atendido con justicia y equidad en el ámbito judicial en función al reconocimiento de los derechos lingüísticos y de la comunicación forense (Bermúdez-Tapia, 2013).

d. Ejercer el derecho a un intérprete cuando se tiene por propio un idioma ajeno al castellano o al ámbito detallado en el artículo $48^{\circ}$ de la Constitución de 1993. En este punto, basta con evaluar la jurisprudencia constitucional detallada en la STC No 00099-2010PHC/TC (Tribunal Constitucional, 2010).

e. Ejercer el derecho a mantener una comunicación eficaz e idónea con alguna persona, entidad o administración pública, en el ámbito comercial, social o económico. En este sentido, la regulación de las "fichas de traducción" en los productos importados que son "etiquetados"en el idioma del país productor deben ser"traducidos" para que así puedan ser distribuidos en el país, regulación detallada en el Decreto Legislativo № 1304 del año 2016 que aprueba la Ley de Etiquetado y Verificación de los Reglamentos Técnicos de los Productos Industriales Manufacturados.

f. Exigir la obligación a las entidades públicas de establecer mecanismos lingüísticos para comunicarse con los ciudadanos. En este sentido, tanto el Poder Judicial, el Ministerio Público, la Policía Nacional del Perú, la SUNAT y otras entidades estatales han venido desarrollando procesos normativos y administrativos que han "establecido" protocolos y "procedimientos" en idiomas originarios, como el quechua y el aimara, sobre todo.

Sin embargo, observamos que no hay una correcta relación en la Política de Estado detallada en los artículos 48, $17^{\circ}$ y $2.19^{\circ}$ de la Constitución de 1993, principalmente porque no se ha tomado en cuenta el valor social, cultural, histórico, político y económico de ampliar la difusión de las lenguas originarias. 


\section{La política de Estado que valora al idioma como elemento productivo en lo cultural}

Frente a lo detallado en el último párrafo del punto precedente, consideramos oportuno iniciar el análisis de nuestra propuesta, sobre la base de la explicación de las diferencias entre "Políticas de Estado" y las "Políticas Públicas".

a. Las Políticas de Estado son aquellas líneas programáticas, consensuadas y democráticamente sostenibles en el tiempo que están detalladas en la Constitución.

En el caso del presente texto, se observa que la Política de Estado está contenido en los artículos 48 y 17º de la Constitución de 1993.

Esto porque en ambos artículos se detalla la importancia del "idioma" en la preservación, desarrollo y difusión de los idiomas originarios para permitir la tutela de derechos de las poblaciones vernáculo hablantes (en el ámbito colectivo y difuso) y la preservación de la identidad étnico cultural, conforme al artículo $2.19^{\circ}$ de la Constitución.

b. Las políticas públicas son aquellas acciones que se caracterizan por ser sectoriales, estructurales y focalizadas en asuntos puntuales y particulares. En este ámbito, son políticas programadas por los Gobiernos Nacionales para tener un objetivo específico, las cuales pueden ser complementarias entre sí pero que procuran atender "puntos delimitados en función a sus acciones de gobierno".

Sobre esta explicación, entonces corresponde detallar que desde 1993, los Gobiernos Nacionales (en el ámbito del Poder Ejecutivo) no han logrado materializar una Política de Estado que pueda promover las políticas públicas de atención a las poblaciones originarias y mestizas del país, generándose como elementos negativos:

a. La evaluación de acciones focalizadas e incongruentes con el ideal nacional de promover una única identidad nacional sin excluir valores socio-culturales e históricos de las poblaciones originarias.

b. La disfuncionalidad del gasto presupuestal nacional en particular porque las acciones ejecutadas no logran atender un objetivo mayor que es la búsqueda de un desarrollo progresivo y sostenible a toda la población nacional, situación que permite el mantenimiento de prácticas sociales discriminatorias y el registro de actos de racismo en la población nacional. 
En este punto, detallamos que históricamente el país no ha logrado "superar" el contexto social, cultural y económico de la Colonia durante la República, principalmente a consecuencia de la coexistencia de las "Repúblicas de Criollos y de Indios", las cuales son expresiones de un nivel de antagonismo social inaceptable en la actualidad (BermúdezTapia, 2019, p. 137 y ss.).

c. La inviabilidad de un proyecto de "país" que se desarrolle en función a una identidad nacional única, donde puedan reconocerse las diferencias étnicas, sociales y culturales como un valor proactivo y provechoso para toda la población nacional.

Es bajo este marco de evaluación que la materialización de una Política de Estado basado en la promoción de los derechos lingüísticos puede detallar una vinculación proactiva en la tutela, promoción y ampliación de los derechos de aquellas comunidades históricamente limitadas en el país, generándose una paulatina inclusión social la cual podría atenuar el severo problema de violencia social que registra nuestro país en la actualidad.

\section{Conclusiones}

Sobre la base del análisis de la normatividad constitucional y de la realidad histórica nacional es posible detallar que en los diferentes Gobiernos Nacionales no se ha tomado en cuenta el carácter excluyente y dividido de la población nacional. Esto ha constituido una referencia negativa que impide la materialización de un proyecto de país progresista, moderno y democráticamente sostenible en el tiempo.

Por tanto, identificar como punto negativo esta realidad permite detallar un panorama que permita proyectar acciones estatales que puedan remediar el actual panorama nacional. Un elemento que viabiliza dicho margen de acciones está detallado en la tutela, preservación, ampliación y mayor difusión de los derechos lingüísticos de las poblaciones originarias y mestizas del país, porque de este modo se podría acceder a la promoción de una inclusión social mucho más eficiente y sostenible.

Igualmente, los efectos positivos de estas acciones podrían mejorar y ampliar el margen cultural e histórico que nuestro país registra, para mejorar cualitativamente como nación y a nivel de las propias poblaciones originarias y/o vernáculo hablantes. 


\section{Referencias bibliográficas}

BERMÚDEZ-TAPIA, Manuel (2000). El idioma materno como derecho. Cathedra Revista de los estudiantes de Derecho de la Universidad Nacional Mayor de San Marcos, Año IV (7), 225-238.

BERMÚDEZ-TAPIA, Manuel (2001). Los derechos lingüísticos. Lima: Asociación no hay derecho.

BERMÚDEZ-TAPIA, Manuel (2001). Categorías de Ciudadanía en el Perú, por el goce de derechos fundamentales. BIRA Boletín del Instituto Riva Agüero (28), 333-343.

BERMÚDEZ-TAPIA, Manuel (2006). Derecho al idioma. Normas Legales (358), 69-73.

BERMÚDEZ-TAPIA, Manuel (2008). La Constitución a través de las Sentencias del Tribunal Constitucional. Lima: Ediciones Legales.

BERMÚDEZ-TAPIA, Manuel (2012). Derecho procesal de familia. Aproximación crítica no convencional a los procesos de familia. Lima: Editorial San Marcos.

BERMÚDEZ-TAPIA, Manuel (2013). Acceso a la justicia a través del lenguaje y comunicación forense, en: ASOCIACIÓN PERUANA DE DERECHO CONSTITUCIONAL. Derecho Constitucional. Lima: ARA.

BERMÚDEZ-TAPIA, Manuel (2018). El acceso al sistema judicial: los derechos lingüísticos como derechos fundamentales. Actualidad Civil (49), 155-163.

BERMÚDEZ-TAPIA, Manuel (2019). Las dos repúblicas en el Perú: indios y criollos de 1821 a 2019. lura 4 (1), 137-146.

BERNALES BALLESTEROS, Enrique. (2013). El desarrollo de la Constitución de 1993 desde su promulgación a la fecha, Pensamiento Constitucional (18), 35-46.

BRAVO TECSI, Fernando (2005). Perú: legado milenario. Lima: Universidad de San Martín de Porres.

HUAYRE COCHACHIN, Rosa (2012). Reforma agraria y comunidades campesinas (Perú 1969-1975): aproximación al proceso de reestructuración comunal. Heraldos: revista de investigación del Centro de Estudiantes de Historia 1 (1), 104-114.

INEI (2010). Perú: estimaciones y proyecciones de población económicamente activa urbana y rural por sexo y grupos de edad, según departamento, 2000-2015. Lima: INEI.

KLEEBERG HIDALGO, Fernando (2001). La industria pesquera en el Perú. Lima: Universidad de Lima. 
LEGIS (2018). iHistórico! Esta es la primera sentencia redactada en aimara en el Perú. Recuperado de https://lpderecho.pe/primera-sentencia-redactada-aimara-peru/

LUNA GONZÁLEZ POLAR, Julio (1964). La casa de gobierno en Lima del libertador Simón Bolívar, 1823-1826. Lima: Librería e imprenta D. Miranda.

MATOS MAR, José (1969). Perú problema. Lima: Moncloa-Campodónico.

OTOYA DE ANDRADE, Elsa (1975). La educación técnica-profesional en el desarrollo socio-económico y cultural del Perú. Tesis para optar por el grado de Doctor en Educación en la PUCP, Lima.

REMY, María. Población indígena y construcción de la democracia en el Perú, 1563. En Ministerio de Cultura (2014), Conflicto y cambios en la sociedad rural. Lima: Ministerio de Cultura.

RUBIO CORREA, Marcial (2017). Para conocer la Constitución de 1993. Lima: PUCP.

SEMINARIO HURTADO, Nuccia. (2019). El derecho fundamental a la educación intercultural bilingüe de los pueblos originarios en el Perú.

SEMINARIO HURTADO, Nuccia \& Rebaza Vílchez, Karen (2018). El derecho a la educación intercultural bilingüe de la niñez indígena en el Perú: avances y desafíos. Persona y Familia 1 (7), 135-163.

TRIBUNAL CONSTITUCIONAL (2010) STC No 00099-2010-PHC/TC. Recuperado de https://www.tc.gob.pe/jurisprudencia/2010/00099-2010-HC\%20Resolucion.html

TRIBUNAL CONSTITUCIONAL (2018) STC No 00889-2017-PA/TC. Recuperado de https://tc.gob.pe/jurisprudencia/2018/00889-2017-AA.pdf 